\title{
Racial disparities in patients diagnosed with light chain (AL) amyloidosis
}

\author{
Anita D'Souza (1)', Liliana Pezzin ${ }^{1,2}$, Purushottam Laud² and Ashima Singh ${ }^{3}$
}

\section{Dear Editor,}

Light chain (AL) amyloidosis arises from a precursor plasma cell neoplasm that produces clonal free light chains that form insoluble fibril deposits leading to organ dysfunction ${ }^{1}$. Because the disease is rare, heterogeneous, and multi-systemic, it can take several months to years for the symptoms to show in patients before a diagnosis is made ${ }^{2}$. Knowledge of the pre-existing clinical characteristics of patients eventually diagnosed with AL amyloidosis is critical as it may inform early diagnosis of the disease. Evidence suggests that early diagnosis of AL amyloidosis leads to improved outcomes, including superior survival, as the disease can be modulated with recently available therapies $^{3,4}$. Among diagnosed amyloidosis patients, Black men and women have the highest mortality rate ${ }^{5}$. Although existing literature has documented a racial predisposition in plasma cell disorders, such as monoclonal gammopathy of undetermined significance (MGUS) and multiple myeloma $^{6,7}$, racial differences in the incidence of the multiple other potential clinical precursor diagnoses associated with AL amyloidosis remain unknown. In this report, we use nationally representative electronic health records (EHR) data to document and contrast the clinical characteristics of patients diagnosed with AL amyloidosis by self-reported race. We also compare the clinical characteristics between patients with AL amyloidosis and matched individuals without amyloidosis by race. We hypothesized that differences in AL-associated, preexisting diagnoses will be evident in patients prior to their diagnosis of AL amyloidosis and that these differences will vary by the patients' race.

\footnotetext{
Correspondence: Anita D'Souza (andsouza@mcw.edu)

'Department of Medicine, Medical College of Wisconsin, Milwaukee, WI 53226, USA

${ }^{2}$ Institute for Health and Equity, Medical College of Wisconsin, Milwaukee, WI 53226, USA
}

Full list of author information is available at the end of the article
Data for this analysis were drawn from TriNetX, a health research network providing access to high-quality de-identified patient-level data from EHR from large healthcare organizations. These data, which are refreshed on a regular basis, are made available through a research network that provides a HIPAA compliant platform with a built-for-purpose user interface and analytics capabilities. No protected health information or personal data is made available to the users of the platform. For this analysis, the TriNetX platform with browser-based realtime analytical features was used.

Patient population: Patients were coded to have AL amyloidosis if they (i) had two or more occurrences of diagnosis codes ICD10: E85.81, E85.4, E85.89, or E85.9 between Jan 1, 2010 and Dec 31, 2019 and (ii) received specific treatment after their AL amyloidosis diagnosis. Specific treatment included the use of one or more of the following: bendamustine, bortezomib, carfilzomib, cyclophosphamide, daratumumab, dexamethasone, doxorubicin, elotuzumab, etoposide, interferon alpha- $2 \mathrm{a} / 2 \mathrm{~b}$, ixazomib, lenalidomide, melphalan, panobinostat, pomalidomide, prednisone, prednisolone, thalidomide, vincristine, or transfusion of autologous hematopoietic cells. The sample was further restricted to individuals aged 40 or older due to the low incidence of the disease in younger adults. The cohort of AL amyloidosis patients was then stratified by race as African American/Black and White. The two comparison groups of Black and White patients without amyloidosis comprised individuals who (i) did not have any ICD code for AL diagnoses and (ii) had at least two visits with the healthcare system in the time period of Jan 1, 2010-Dec 31, 2019. To ensure the comparability of the comparison groups, we further restricted the cohorts to those who had at least one visit in 2019 .

Pre-existing diagnoses were defined using diagnostic codes of interest present in an $\mathrm{AL}$ amyloidosis patient prior to the diagnosis of AL amyloidosis. These were 
grouped within four major categories: cardiac, renal, gastrointestinal/hepatic, other. For example, the cardiac category included diagnostic codes for cardiomyopathy, heart failure, cardiac arrhythmias, etc. The specific codes considered are provided in the tables.

Statistical analyses: We used $z$-tests/t-tests to compare the clinical characteristics of Black and White AL amyloidosis patients after matching for age, sex, and presence of type 2 diabetes. We matched with diabetes as some ALassociated diagnoses, e.g. cardiomyopathy, heart failure, proteinuria, chronic kidney disease, and neuropathy, may overlap with diabetes and there is a known racial difference in the prevalence of diabetic complications. We performed 1:1 matching based on the greedy nearest-neighbor matching algorithm using caliper of 0.1 pooled standard deviations (SD). The same methodology was used to compare the preexisting clinical characteristics of patients with and without a diagnosis of AL amyloidosis by race.

A total of 4028 patients were identified as having a diagnosis of $\mathrm{AL}$ amyloidosis during the study period. Among Black patients with AL amyloidosis $(N=695)$, the mean age was 65 (SD 11) years with 55\% males. The geographic distribution included 16\% Northeast, 23\% Midwest, 56\% South, and 5\% West. Among 3333 identified White patients, the mean age was 67 years (SD 11) with $58 \%$ male. The geographic distribution included $17 \%$ Northeast, 24\% Midwest, 41\% South, 17\% West, and 1\% unknown. The age difference by race appeared smaller in AL compared to MGUS ${ }^{8}$. After 1:1 matching, 691 Black AL patients matched with 691 White AL patients.

Compared to White AL patients, Black patients were more likely to have pre-existing MGUS as well as multiple other cardiac, renal, gastrointestinal AL-associated diagnoses, and carpal tunnel syndrome preceding the formal diagnosis of AL amyloidosis (Table 1). The difference in pre-existing MGUS was different (21\% Black vs $13 \%$ White, $p 4.48 \mathrm{E}-05)$ but pre-existing multiple myeloma was not different between the two groups (32.4\% Black vs $30.83 \%$ White, $p$ 0.53). This may suggest that once multiple myeloma is diagnosed, patients are in the care of specialists who may be vigilant for identification of amyloidosis and there is no racial difference in this diagnosis. The magnitude of differences was particularly striking for the diagnoses of cardiomegaly $(28.51 \%$ vs $20.55 \%$, $p$ 0.0006), cardiomyopathy ( $37.19 \%$ vs $22.58 \%, p 2.93 \mathrm{E}$ $-09)$, heart failure $(54.85 \%$ vs $33.29 \%, p 6.85 \mathrm{E}-16)$, and chronic kidney disease $(43.56 \%$ vs $35.02 \%, p$ 0.001), for Black compared to White patients, respectively. These clinical conditions are known harbingers of worse outcomes in AL amyloidosis. These findings raise concerns that Black patients with AL amyloidosis may be diagnosed later in the disease process. These findings also suggest that it may take longer or require a greater constellation of $\mathrm{AL}$-associated symptoms/diagnoses before $\mathrm{AL}$ amyloidosis to be diagnosed in Black patients relative to White individuals. It is well known that patients with $\mathrm{AL}$ see multiple physicians including subspecialists for various diagnoses, e.g., proteinuria, carpal tunnel syndrome, cardiomyopathy, related to the amyloidosis diagnosis ${ }^{2,9}$, and our results suggest that race can be associated with AL amyloidosis diagnosis.

To shed further light on the racial differences in AL amyloidosis, we examined the extent to which preexisting diagnoses differ by race among patients with and without AL amyloidosis. Table 2 shows the results of this analysis applied to samples matched by age, sex, and presence of type 2 diabetes, which yielded 695 Black patients and 3333 White patients without AL amyloidosis. As expected, there was a significantly higher incidence of MGUS, as well as most other AL-associated organ-specific and other pre-existing diagnoses of interest preceding the diagnosis of $\mathrm{AL}$ amyloidosis within each racial group, the prevalence of which was considerably higher among Black patients with AL. This pattern confirms the notion that AL patients have a different clinical profile than those who do not develop the disease. These findings also suggest that it may be possible to develop an early warning system through advanced statistical models that would identify patients at risk for developing AL amyloidosis earlier in the disease course. Additionally, there was a higher prevalence of AL-associated pre-existing conditions among the subgroup of African American/ Black individuals without $\mathrm{AL}$ when compared to their White counterparts, pointing to a higher burden of comorbidities or a higher proportion of undiagnosed AL amyloidosis in Black individuals compared to Whites. Staron, et al. recently reported that among patients seen at an Amyloid Center of Excellence, Black patients had a more aggressive phenotype ${ }^{10}$. Additionally, other systemic barriers and social determinants of health may further result in delays/exclusion of AL amyloidosis patients being referred to centers of excellence, thus resulting in additional race-based disparities in AL amyloidosis care, which needs to be further explored.

Our study is limited by the lack of granular data on AL amyloidosis, including the lack of information on stage and biomarkers. Nonetheless, these large, nationwide EHR data provide a valuable opportunity to understand the clinical burden of AL amyloidosis. These data also suggest that $\mathrm{AL}$ is not as rare even with our conservative definition using diagnostic codes. Our findings are consistent with the hypothesis that Black patients may experience under- or delayed diagnosis, as suggested by their higher burden of pre-existing, AL-associated diagnoses prior to the formal AL diagnosis. This is especially true for critical organ conditions associated with early mortality. Finally, there are significant differences, overall and across racial groups, in the prevalence of pre-existing 
Table 1 Comparison of pre-existing diagnoses in AL amyloidosis by race.

\begin{tabular}{llllr}
\hline ICD code & Pre-existing diagnosis & Black, with AL $\boldsymbol{N}=\mathbf{6 9 1}$ & White, with AL $\boldsymbol{N}=\mathbf{6 9 1}$ & $\boldsymbol{p}$-value \\
\hline D47.2 & MGUS & $21.13 \%$ & $12.88 \%$ & $4.48 \mathrm{E}-05$ \\
C90 & Multiple myeloma & $32.42 \%$ & $30.83 \%$ & 0.22 \\
\end{tabular}

Cardiac diagnoses

$\begin{array}{lll}\text { 151.7 } & \text { Cardiomegaly } & 28.51 \% \\ 142 & \text { Cardiomyopathy } & 37.19 \% \\ \text { I49 } & \text { Other cardiac arrythmia } & 30.25 \% \\ \text { R06.0 } & \text { Dyspnea } & 49.78 \% \\ \text { R55 } & \text { Syncope and collapse } & 13.46 \% \\ \text { R42 } & \text { Dizziness and giddiness } & 14.33 \% \\ \text { R60 } & \text { Edema } & 33.58 \% \\ 150 & \text { Heart failure } & 54.85 \% \\ \text { J90 } & \text { Pleural effusion } & 11.43 \% \\ \text { I48 } & \text { Atrial fibrillation and flutter } & 21.85 \% \\ 195 & \text { Hypotension } & 21.71 \%\end{array}$

Renal diagnoses

N04, Nephrotic syndrome $\quad 8.39 \%$

R80 Proteinuria $23.16 \%$

N18 Chronic kidney disease

\section{Neurological diagnoses}

$\begin{array}{ll}\text { N52.9 } & \text { Male erectile dysfunction } \\ \text { M79.2 } & \text { Neuralgia and neuritis } \\ \text { R20 } & \text { Disturbances of skin sensations } \\ \text { G60 } & \text { Hereditary and idiopathic neuropathy } \\ \text { G62 } & \text { Other and unspecified polyneuropathies } \\ \text { G90 } & \text { Disorders of autonomic nervous system }\end{array}$

\section{GI/Hepatic diagnoses}

$\begin{array}{ll}\text { R13.1 } & \text { Dysphagia } \\ \text { R16 } & \text { Hepatomegaly and splenomegaly } \\ \text { K76 } & \text { Other diseases of liver } \\ \text { R11 } & \text { Nausea and vomiting } \\ \text { K59.0 } & \text { Constipation } \\ \text { R19.7 } & \text { Diarrhea } \\ \text { R10 } & \text { Abdominal pain }\end{array}$

Other diagnoses

$\begin{array}{ll}\text { R53 } & \text { Malaise and fatigue } \\ \text { G56.0 } & \text { Carpal Tunnel Syndrome } \\ \text { D69 } & \text { Purpura } \\ \text { Q38 } & \text { Macroglossia }\end{array}$

Patients matched by age, sex, and presence of diabetes mellitus. $S D$ standard difference.

\begin{tabular}{llll} 
Black, with AL $\boldsymbol{N}=\mathbf{6 9 1}$ & White, with AL $\mathbf{N}=\mathbf{6 9 1}$ & $\boldsymbol{p}$-value & SD \\
\hline $21.13 \%$ & $12.88 \%$ & $4.48 \mathrm{E}-05$ & 0.22 \\
$32.42 \%$ & $30.83 \%$ & 0.53 & 0.03 \\
& & & \\
$28.51 \%$ & $20.55 \%$ & 0.0006 & 0.19 \\
$37.19 \%$ & $22.58 \%$ & $2.93 \mathrm{E}-09$ & 0.32 \\
$30.25 \%$ & $19.68 \%$ & $5.7 \mathrm{E}-06$ & 0.25 \\
$49.78 \%$ & $35.17 \%$ & $3.88 \mathrm{E}-08$ & 0.30 \\
$13.46 \%$ & $9.70 \%$ & 0.03 & 0.12 \\
$14.33 \%$ & $14.62 \%$ & 0.88 & 0.01 \\
$33.58 \%$ & $26.77 \%$ & 0.006 & 0.15 \\
$54.85 \%$ & $33.29 \%$ & $6.85 \mathrm{E}-16$ & 0.45 \\
$11.43 \%$ & $11.72 \%$ & 0.87 & 0.01 \\
$21.85 \%$ & $21.85 \%$ & 1 & 0 \\
$21.71 \%$ & $18.67 \%$ & 0.16 & 0.08
\end{tabular}

$43.56 \%$

$9.84 \%$

0.35

0.05

$19.97 \%$

0.15

0.08

$35.02 \%$

0.001

$$
7.67 \%
$$

$12.88 \%$

$5.79 \%$

$13.17 \%$

$19.83 \%$

$22.00 \%$

$15.63 \%$

$27.50 \%$

$30.83 \%$

$11.14 \%$

$15.20 \%$

$2.32 \%$
$4.34 \%$

$2.61 \%$

$11.00 \%$

$10.42 \%$

$11.29 \%$

$3.47 \%$

0.009

$9.70 \%$

$5.79 \%$

$11.00 \%$

$16.35 \%$

$14.76 \%$

$17.95 \%$

$19.25 \%$

$30.39 \%$

$7.24 \%$

$14.47 \%$

$1.45 \%$
0.60

0.10

0.21

0.80

0.44

0.14

0.01

0

0.07

0.22

0.09

0.09

0.19

0.25

0.06

0.0003

0.20

$0.86 \quad 0.01$

$0.01 \quad 0.14$

$0.71 \quad 0.02$

0.24

0.06 
Table 2 Comparison between pre-existing diagnoses in individuals with or without AL amyloidosis stratified by race.

\begin{tabular}{|c|c|c|c|c|c|c|c|c|c|}
\hline ICD10 code & Pre-existing diagnosis & $\begin{array}{l}\text { Black, with } \\
\text { AL } N=695\end{array}$ & $\begin{array}{l}\text { Black, no } \\
\text { AL } N=695\end{array}$ & $\begin{array}{l}p \text {-value Black, } \\
\text { AL vs not }\end{array}$ & SD & $\begin{array}{l}\text { White, with } \\
\text { AL } N=3333\end{array}$ & $\begin{array}{l}\text { White, no } \\
\text { AL } N=3333\end{array}$ & $\begin{array}{l}p \text {-value White, } \\
\text { AL vs not }\end{array}$ & SD \\
\hline D47.2 & MGUS & $21.01 \%$ & $1.44 \%$ & $6.84 \mathrm{E}-31$ & 0.65 & $16.41 \%$ & $0.36 \%$ & 0 & 0.61 \\
\hline C90 & Multiple myeloma & $32.23 \%$ & $1.44 \%$ & 0 & 0.90 & $32.67 \%$ & $0.30 \%$ & 0 & 0.97 \\
\hline \multicolumn{10}{|c|}{ Cardiac diagnoses } \\
\hline 151.7 & Cardiomegaly & $28.92 \%$ & $9.35 \%$ & $1.81 \mathrm{E}-20$ & 0.51 & $20.13 \%$ & $4.08 \%$ & 0 & 0.51 \\
\hline 142 & Cardiomyopathy & $37.41 \%$ & $5.04 \%$ & 0 & 0.86 & $23.19 \%$ & $2.70 \%$ & 0 & 0.64 \\
\hline 149 & Other cardiac arrhythmia & $30.50 \%$ & $10.50 \%$ & $2.59 \mathrm{E}-20$ & 0.51 & $22.05 \%$ & $8.16 \%$ & 0 & 0.40 \\
\hline R06.0 & Dyspnea & $50.07 \%$ & $21.73 \%$ & $3.24 \mathrm{E}-28$ & 0.62 & $38.52 \%$ & $15.00 \%$ & 0 & 0.55 \\
\hline R55 & Syncope and collapse & $13.53 \%$ & $7.05 \%$ & 7.1E-05 & 0.21 & $10.59 \%$ & $3.93 \%$ & $1.08 \mathrm{E}-25$ & 0.26 \\
\hline R42 & Dizziness and giddiness & $14.53 \%$ & $13.09 \%$ & 0.43694 & 0.04 & $15.51 \%$ & $9.57 \%$ & $2.43 E-13$ & 0.18 \\
\hline R60 & Edema & $33.67 \%$ & $12.95 \%$ & $6.53 \mathrm{E}-20$ & 0.51 & $27.69 \%$ & $7.56 \%$ & 0 & 0.55 \\
\hline 150 & Heart failure & $54.96 \%$ & $11.94 \%$ & 0 & 1.03 & $33.00 \%$ & $5.88 \%$ & 0 & 0.73 \\
\hline J90 & Pleural Effusion & $11.66 \%$ & $2.16 \%$ & $2.92 \mathrm{E}-12$ & 0.38 & $10.08 \%$ & $1.26 \%$ & 0 & 0.39 \\
\hline 148 & Atrial fibrillation and flutter & $22.01 \%$ & $6.04 \%$ & $1.01 \mathrm{E}-17$ & 0.47 & $21.84 \%$ & $8.40 \%$ & 0 & 0.38 \\
\hline 195 & Hypotension & $22.01 \%$ & $6.33 \%$ & $5.18 \mathrm{E}-17$ & 0.46 & $17.91 \%$ & $3.57 \%$ & 0 & 0.48 \\
\hline \multicolumn{10}{|c|}{ Renal diagnoses } \\
\hline N04 & Nephrotic syndrome & $8.35 \%$ & $1.44 \%$ & $2.39 \mathrm{E}-09$ & 0.32 & $10.08 \%$ & $0.30 \%$ & 0 & 0.45 \\
\hline R80 & Proteinuria & $23.31 \%$ & $4.17 \%$ & $3.70 E-25$ & 0.58 & $19.92 \%$ & $2.13 \%$ & 0 & 0.59 \\
\hline N18 & Chronic Kidney disease & $43.89 \%$ & $14.53 \%$ & $2.38 \mathrm{E}-33$ & 0.68 & $33.15 \%$ & $6.48 \%$ & 0 & 0.71 \\
\hline \multicolumn{10}{|c|}{ Neurological diagnoses } \\
\hline N52.9 & Male erectile dysfunction & $7.63 \%$ & $9.07 \%$ & 0.332133 & 0.05 & $4.50 \%$ & $4.71 \%$ & 0.68 & 0.01 \\
\hline M79.2 & Neuralgia and Neuritis & $2.30 \%$ & $3.31 \%$ & 0.255555 & 0.06 & $3 \%$ & $1.29 \%$ & $1.45 \mathrm{E}-06$ & 0.12 \\
\hline R20 & $\begin{array}{l}\text { Disturbances of skin } \\
\text { sensations }\end{array}$ & $13.96 \%$ & $8.49 \%$ & 0.001 & 0.17 & $14.13 \%$ & $7.26 \%$ & $1.13 E-19$ & 0.22 \\
\hline G60 & $\begin{array}{l}\text { Hereditary and idiopathic } \\
\text { neuropathy }\end{array}$ & $12.81 \%$ & $3.02 \%$ & $1.41 \mathrm{E}-11$ & 0.37 & $10.77 \%$ & $2.10 \%$ & 0 & 0.36 \\
\hline G62 & $\begin{array}{l}\text { Other and unspecified } \\
\text { polyneuropathies }\end{array}$ & $11.66 \%$ & $4.17 \%$ & $2.38 \mathrm{E}-07$ & 0.28 & $12.51 \%$ & $3.78 \%$ & $8.26 \mathrm{E}-39$ & 0.32 \\
\hline G90 & $\begin{array}{l}\text { Disorders of autonomic } \\
\text { nervous system }\end{array}$ & $2.73 \%$ & $1.44 \%$ & 0.091225 & 0.09 & $3.24 \%$ & $0.30 \%$ & $8.82 \mathrm{E}-20$ & 0.22 \\
\hline \multicolumn{10}{|l|}{ GI/hepatic } \\
\hline R13.1 & Dysphagia & $12.81 \%$ & $6.19 \%$ & $2.57 \mathrm{E}-05$ & 0.23 & $9.84 \%$ & $5.19 \%$ & $5.99 \mathrm{E}-13$ & 0.18 \\
\hline R16 & $\begin{array}{l}\text { Hepatomegaly and } \\
\text { splenomegaly }\end{array}$ & $5.76 \%$ & $1.73 \%$ & 7.57E-05 & 0.21 & $4.35 \%$ & $1.56 \%$ & $1.74 \mathrm{E}-11$ & 0.17 \\
\hline K76 & Other diseases of liver & $13.38 \%$ & $6.91 \%$ & $6.39 \mathrm{E}-05$ & 0.22 & $9.15 \%$ & $4.56 \%$ & $1.21 \mathrm{E}-13$ & 0.18 \\
\hline R11 & Nausea and vomiting & $20 \%$ & $11.66 \%$ & $2.02 \mathrm{E}-05$ & 0.23 & $15.51 \%$ & $7.29 \%$ & 4.61E-26 & 0.26 \\
\hline K59.0 & Constipation & $22.16 \%$ & $12.81 \%$ & $4.43 \mathrm{E}-06$ & 0.25 & $14.67 \%$ & $6.45 \%$ & $9.31 \mathrm{E}-28$ & 0.27 \\
\hline R19.7 & Diarrhea & $15.54 \%$ & $6.76 \%$ & $2.01 E-07$ & 0.28 & $15.27 \%$ & $6.90 \%$ & $1.37 \mathrm{E}-27$ & 0.27 \\
\hline R10 & Abdominal pain & $27.63 \%$ & $24.60 \%$ & 0.20 & 0.07 & $20.16 \%$ & $17.01 \%$ & 0.001 & 0.08 \\
\hline
\end{tabular}


Table 2 continued

\begin{tabular}{|c|c|c|c|c|c|c|c|c|c|}
\hline ICD10 code & Pre-existing diagnosis & $\begin{array}{l}\text { Black, with } \\
\text { AL } N=695\end{array}$ & $\begin{array}{l}\text { Black, no } \\
\text { AL } N=695\end{array}$ & $\begin{array}{l}p \text {-value Black, } \\
\text { AL vs not }\end{array}$ & SD & $\begin{array}{l}\text { White, with } \\
\text { AL } N=3333\end{array}$ & $\begin{array}{l}\text { White, no } \\
\text { AL } N=3333\end{array}$ & $\begin{array}{l}p \text {-value White, } \\
\text { AL vs not }\end{array}$ & SD \\
\hline \multicolumn{10}{|l|}{ Other } \\
\hline R53 & Malaise and fatigue & $30.79 \%$ & $18.56 \%$ & $1.24 \mathrm{E}-07$ & 0.29 & $32.73 \%$ & $15.12 \%$ & 0 & 0.42 \\
\hline G56.0 & Carpal Tunnel Syndrome & $11.08 \%$ & $4.17 \%$ & $1.23 \mathrm{E}-06$ & 0.26 & $8.34 \%$ & $3.36 \%$ & 4.60E-18 & 0.21 \\
\hline D69 & Purpura & $15.25 \%$ & $3.45 \%$ & $4.23 \mathrm{E}-14$ & 0.41 & $12.90 \%$ & $2.82 \%$ & 0 & 0.38 \\
\hline K14.8 & Macroglossia & $2.30 \%$ & $0 \%$ & $5.74 \mathrm{E}-05$ & 0.22 & $1.44 \%$ & $0.30 \%$ & $5.40 \mathrm{E}-07$ & 0.12 \\
\hline
\end{tabular}

Patients matched by age, sex, and presence of diabetes mellitus. $S D$ standard difference.

diagnoses in individuals with and without AL, suggesting the feasibility and value of developing predictive algorithms aimed at identifying patterns of precursor conditions associated with the likelihood of an AL amyloidosis diagnosis. These results highlight significant racial disparities in $\mathrm{AL}$ amyloidosis diagnosis. Our next steps include studying differences in the length of time for preexisting diagnoses prior to AL diagnosis as well outcomes after the diagnosis, including the burden of illness. Future work is also needed to study the causes and consequences of racial disparities on the disease course and mortality of AL amyloidosis patients.

\section{Author details}

'Department of Medicine, Medical College of Wisconsin, Milwaukee, WI 53226, USA. ${ }^{2}$ Institute for Health and Equity, Medical College of Wisconsin, Milwaukee, WI 53226, USA. ${ }^{3}$ Department of Pediatrics, Medical College of Wisconsin, Milwaukee, WI 53226, USA

\section{Author contributions}

All authors contributed toward study design, analysis, review of the findings, and manuscript writing.

\section{Conflict of interest}

The authors declare no competing interests. Research reported in this publication was supported by K23 HL141445 (AD). The content is solely the responsibility of the authors and does not necessarily represent the official views of the National Institutes of Health. The TriNetX data reported here have been supplied by the Medical College of Wisconsin Clinical Research Data Warehouse. The interpretation and reporting of these data are the responsibility of the author(s) and in no way should be seen as an official policy of or interpretation by the Medical College of Wisconsin.

\section{Publisher's note}

Springer Nature remains neutral with regard to jurisdictional claims in published maps and institutional affiliations.

Received: 8 February 2021 Revised: 15 March 2021 Accepted: 25 March 2021

Published online: 10 April 2021

\section{References}

1. Merlini, G. AL amyloidosis: from molecular mechanisms to targeted therapies. Hematology 2017, 1-12 (2017).

2. Lousada, I., Comenzo, R. L., Landau, H., Guthrie, S. \& Merlini, G. Light chain amyloidosis: patient experience survey from the Amyloidosis Research Consortium. Adv. Ther. 32, 920-928 (2015).

3. Kumar, S. et al. Revised prognostic staging system for light chain amyloidosis incorporating cardiac biomarkers and serum free light chain measurements. J. Clin. Oncol. 30, 989-995 (2012).

4. Merlini, G. \& Palladini, G. Treating advanced cardiac damage in light chain amyloidosis: still an unmet need. Haematologica 99, 1407-1409 (2014).

5. Alexander, K. M. et al. Geographic disparities in reported US amyloidosis mortality from 1979 to 2015: potential underdetection of cardiac amyloidosis. JAMA Cardi 3, 865-870 (2018).

6. Landgren, $\mathrm{O}$. et al. Risk of monoclonal gammopathy of undetermined significance (MGUS) and subsequent multiple myeloma among African American and white veterans in the United States. Blood 107, 904-906 (2006).

7. Landgren, $\mathrm{O}$. et al. Prevalence of myeloma precursor state monoclonal gammopathy of undetermined significance in 12372 individuals 10-49 years old: a population-based study from the National Health and Nutrition Examination Survey. Blood Cancer J. 7, e618 (2017).

8. Greenberg, A. J., Vachon, C. M. \& Rajkumar, S. V. Disparities in the prevalence, pathogenesis and progression of monoclonal gammopathy of undetermined significance and multiple myeloma between blacks and whites. Leukemia 26, 609-614 (2012).

9. McCausland, K. L. et al. Light chain (AL) amyloidosis: The journey to diagnosis. Patient 11, 207-216 (2018).

10. Staron, A., Connors, L. H., Zheng, L., Doros, G. \& Sanchorawala, V. Race/ethnicity in systemic AL amyloidosis: perspectives on disease and outcome disparities. Blood Cancer J. 10, 118 (2020). 